\title{
A Population-based Study of Peripartum Cardiomyopathy in Southern Israel: Are Bedouin Women a New High-risk Group?
}

\author{
Louise Kezerle, M.D. ${ }^{1}$, Iftach Sagy, M.D. ${ }^{1,2 *}$, Leah Shalev, M.D. ${ }^{1}$, Offer Erez, \\ M.D. ${ }^{3}$, and Leonid Barski, M.D. ${ }^{1}$ \\ ${ }^{\prime}$ Department of Internal Medicine "F," Soroka University Medical Center, Beersheva, Israel; ${ }^{2}$ Clinical \\ Research Center, Soroka University Medical Center, Beersheva, Israel; and ${ }^{3}$ Department of Obstetrics "D," \\ Soroka University Medical Center, Beersheva, Israel
}

\begin{abstract}
Objectives: Peripartum cardiomyopathy (PPCM) is a serious complication of pregnancy. Studies investigating the risk factors that worsen outcomes have yielded conflicting results. The goals of this study were to describe the clinical and echocardiographic characteristics of PPCM in a single tertiary center and to determine the prognostic factors associated with persistence of left ventricular (LV) dysfunction in these women.

Study Design: This retrospective cross-sectional population-based cohort study included all patients with PPCM confirmed by echocardiography who delivered at our center from 2004 to 2014. Two groups were compared to determine long-term maternal outcome: (1) those who recovered normal LV function; and (2) those with residual systolic LV dysfunction.
\end{abstract}

\footnotetext{
Abbreviations: echo, echocardiogram; EF, ejection fraction; ESC, European Society of Cardiology; HF, heart failure; HTN, hypertension; LV, left ventricular; LVEF, left ventricular ejection fraction; NYHA, New York Heart Association; PPCM, peripartum cardiomyopathy; RV, right ventricular.

Citation: Kezerle L, Sagy I, Shalev L, Erez O, Barski L. A Population-based Study of Peripartum Cardiomyopathy in Southern Israel: Are Bedouin Women a New High-risk Group? Rambam Maimonides Med J 2018;9 (2):eoo11. doi:10.5041/RMMJ.10331

Copyright: (C) 2018 Kezerle et al. This is an open-access article. All its content, except where otherwise noted, is distributed under the terms of the Creative Commons Attribution License (http://creativecommons.org/licenses/by/3.0), which permits unrestricted use, distribution, and reproduction in any medium, provided the original work is properly cited.

Conflict of interest: No potential conflict of interest relevant to this article was reported.

* To whom correspondence should be addressed. E-mail: iftachsagy@gmail.com
} 
Results: There were 148,994 deliveries during the study period. Of these, 89,196 patients were Bedouin and 59,798 were non-Bedouin. Forty-six patients met the PPCM study inclusion criteria. The PPCM prevalence for the total deliveries was 1:3,239. The PPCM prevalence among Bedouin patients was 1:2,787 versus non-Bedouin patients of 1:4,983 $(P=0.037)$. None of the women had pre-existing chronic hypertension, and there was no maternal death. Patients who had severe or moderate LV dysfunction at the clinical presentation of PPCM were less likely to regain normal LV function than those with mild dysfunction (81.2\% versus $56.7 \%, P=0.009$ ). Based on initial echocardiogram, a trend toward residual LV dysfunction was noted in patients with a dilated left ventricle as compared to those with a non-dilated left ventricle (18.8\% versus $6.7 \%, P=0.32$ ). A hypokinetic right ventricle was found in $15.2 \%$ of the women who suffered from PPCM.

Conclusion: In our cohort, Bedouin women may be at increased risk for PPCM, and patients with severe LV dysfunction have a lower chance of recovery from PPCM.

KEY WORDS: Epidemiology, peripartum cardiomyopathy, prognostic factors

\section{INTRODUCTION}

Pregnancy and childbirth pose a great challenge to the maternal cardiovascular system. The maternal circulation changes physiologically during pregnancy but gradually returns to its non-pregnancy state during puerperium. Peripartum cardiomyopathy (PPCM), however, refers to the development of clinical heart failure with reduced left ventricular ejection fraction (LVEF) in the final stages of pregnancy or in the early period after delivery. ${ }^{1}$ It affects previously healthy women, and its etiology is thus far unknown. The available experimental evidence suggests that multiple risk factors contribute to a common final pathway of myocardial damage caused by enhanced oxidative stress, altered prolactin processing, ${ }^{2,3}$ and impaired vascular endothelial growth factor signaling. 4 Several factors have been proposed to contribute to the development of PPCM: older maternal age, 5 African descent, $, 6,7$ hypertensive diseases (chronic hypertension, preeclampsia, and eclampsia), ${ }^{8}$ multi-fetal pregnancy, $, 5,9$ long-term oral tocolytic therapy, ${ }^{10}$ and maternal cocaine abuse. ${ }^{11-13}$

The reported incidence of PPCM varies largely among countries, ranging from 1:300 live births in Africa and Haiti to 1:4,000 live births in the United States.1,14,15 The outcome of PPCM is also highly variable. Some women present a quick clinical improvement and show significant echocardiographic recovery of left ventricular (LV) function, whereas in others the disease progresses to cardiogenic shock, arrhythmias, and even death.5,16-18 Mortality rates of women with PPCM vary from $4 \%$ to up to $19 \%$ in the United States and as high as $28 \%$ in other regions.7,13,18-21 Prognostic factors consistently associ- ated with a higher risk of death are New York Heart Association (NYHA) functional class at presentation, ${ }^{22}$ LVEF $\leq 25 \%-30 \%, 18,23$ black race, ${ }^{16,17,24}$ indigent status, ${ }^{25}$ multiparity, ${ }^{24}$ and age greater than $30 . .^{26,27}$

Conversely, recovery of LV function occurs more frequently in PPCM than with other types of dilated cardiomyopathies, ${ }^{28}$ with $20 \%$ to $60 \%$ of affected women showing complete recovery of LV function in some series. ${ }^{16,18}$ Lower LVEF, larger LV end-diastolic diameter at diagnosis, and black race appear to be adverse predictors for recovery, ${ }^{29-32}$ although there is still uncertainty as to the significance of these findings as parameters that can successfully predict better outcomes in different populations.

The goals of this study were to describe in a population-based cohort the epidemiological, clinical, and obstetrical characteristics of PPCM in southern Israel and to determine the prognostic factors associated with persistence of LV dysfunction in these women.

\section{METHODS}

We conducted a cross-sectional population-based retrospective cohort study. Patients were identified in our electronic database from January 2004 to December 2014 according to the diagnosis of PPCM (ICD-9 674.5) or other cardiovascular diseases complicating pregnancy, childbirth, or puerperium (ICD-9 678.6). The charts of identified patients were reviewed by two independent doctors to determine which ones fulfilled the diagnostic criteria for PPCM according to the 2010 European Society of Cardiology (ESC) Working Group on peripartum 
cardiology ${ }^{14}$ : (1) development of heart failure (HF) toward the end of pregnancy or in the months following delivery; (2) absence of another identifiable cause for the heart failure; and (3) LV systolic dysfunction with a LVEF less than $45 \%$, with the left ventricle being dilated or not dilated. Women with any previous valvular or congenital heart diseases and/or the presence of other severe conditions that justified the reduced cardiac ejection fraction (EF) were excluded. The obstetrical and medical records of patients included in the study were retrieved, and a unified data set was established. As this was a retrospective study, all data were gained from electronic databases only. After patients were identified by their name and official identity card number, each case was randomized and assigned a different study identification number to ensure patient confidentiality. Furthermore, in order to identify possible predictors of poorer recovery, the study cohort was then divided into two groups: (1) those who recovered normal LV function; and (2) those with residual LV dysfunction, moderate or severe. Our institution uses a standardized grading system for $\mathrm{LV}$ dysfunction, in accordance with international values: an $\mathrm{EF} \leq 35 \%$ is diagnosed as severe LV dysfunction; an $\mathrm{EF}$ of $35 \%-45 \%$ is diagnosed as moderate LV dysfunction; and $\mathrm{EF}$ of $45 \%-55 \%$ is considered mild LV dysfunction. Since the numerical estimate of EF was not available for every patient record, the independent cardiologists followed the same qualitative grading system according to these definitions. 33

This study was approved by the ethics committee of the Soroka University Medical Center.

\section{Statistical Analysis}

Theoretic statistics (prevalence and standard deviations) was used for data analysis to describe the demographic, obstetric, and medical characteristics of the population in question. We performed independent $t$ tests and chi-square tests to compare continuous and categorical variables between groups of patients with and without residual LV dysfunction on follow-up echocardiogram (echo). Multivariate analysis was not performed due to the small sample size and the difficulty with achieving statistical significance ( $\alpha$ less than o.1). All the statistical analyses were performed using SPSS 20.0 (IBM, Armonk, NY, USA).

\section{RESULTS}

There were 148,994 deliveries between January 2004 and December 2014 (Bedouin, $n=89,196$; nonBedouin, $n=59,798$ ). Based on ICD-9 diagnosis, 523 patients were identified. The full time period for follow-up was not available. After careful review of echo results, only 46 patients (Bedouin, $n=32$; nonBedouin, $n=14$ ) met all the eligibility criteria and were included in the statistical analysis. Figure 1 provides a flowchart showing the process for selecting the eligible patients.

The overall PPCM prevalence was 1:3,239 births. Of the 46 patients in the study group, 32 were Bedouin and 14 were non-Bedouin. The prevalence of PPCM among Bedouin women was 1:2,787, whereas the PPCM prevalence among non-Bedouin women was $1: 4,983(P=0.037)$. Table 1 presents the baseline maternal and gestational characteristics for the Bedouin and non-Bedouin women. Bedouin patients showed a trend for younger maternal age, had a lower gestational week, and lower birth weight-although these differences did not reach statistical significance. There were no deaths reported among PPCM patients for the period in question.

When comparing the women who had residual LV dysfunction on follow-up echocardiography to those who recovered normal LV systolic function, there were no significant differences in relation to the demographic or obstetric characteristics. Moreover, when comparing other factors previously described as associated with a worse outcome, such as maternal age over 35 and multiparity, no statistical difference was noted between the two groups.

Based on the echo results, the majority of patients had at least moderate LV dysfunction (65.2\%), while $15.2 \%$ also showed signs of right ventricular (RV) compromise. In comparison to the first echo results, $76 \%$ of the patients showed improvement in their LV systolic function on a follow-up echo examination, with $65.2 \%$ regaining normal LV function (Table 2).

When comparing patients with residual PPCM to those without residual PPCM, those with severe and moderate LV dysfunction (13 [81.2\%] versus 17 [56.7\%], respectively) were more likely to have developed residual damage in the second echo exam 


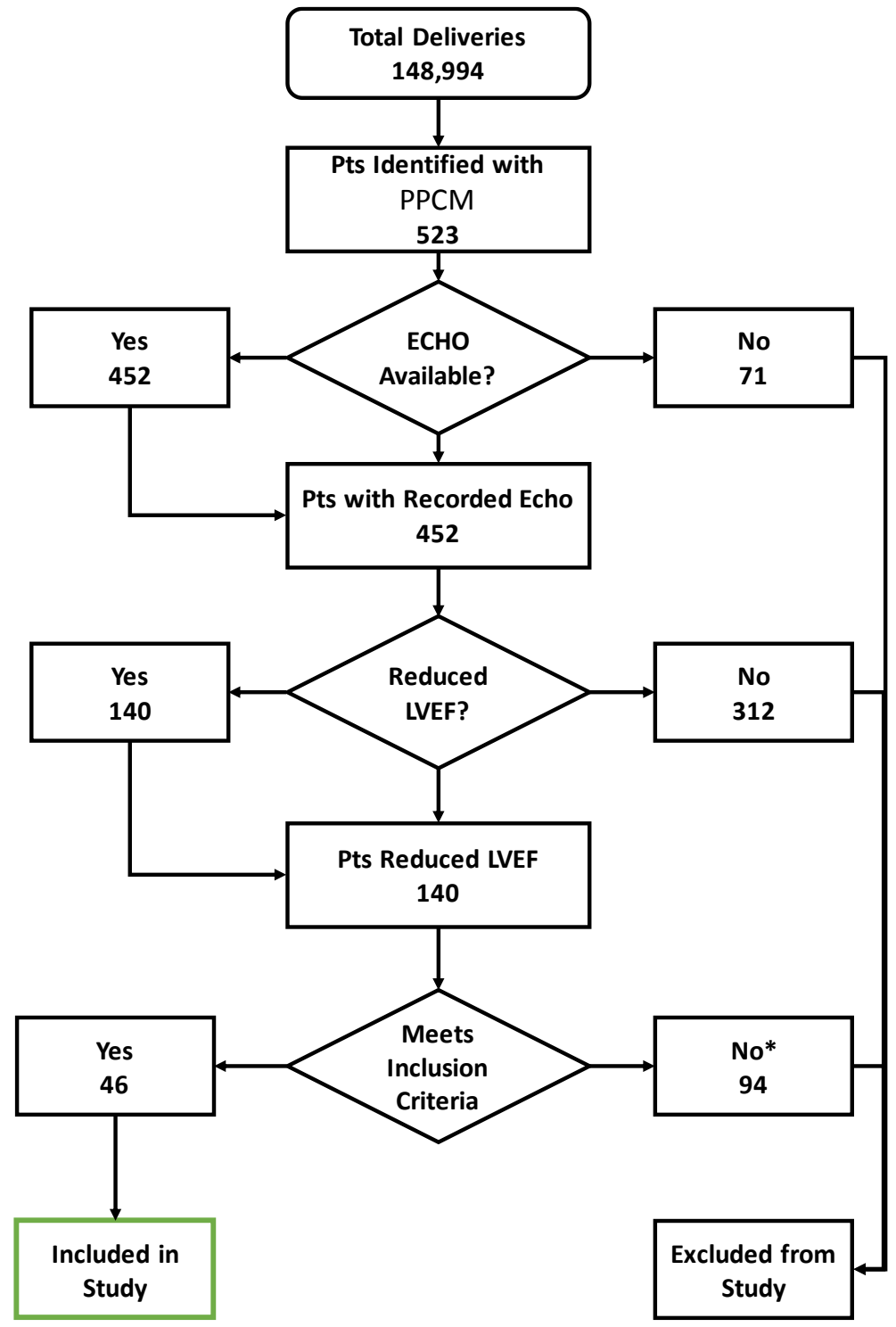

Figure 1. Study Participants Flowchart.

*74 pts with valvular disease; 9 pts with previous cardiomyopathy; 11 pts with other medical conditions. ECHO, echocardiogram; LVEF, left ventricular ejection fraction; PPCM, peripartum cardiomyopathy; Pts, patients.

$(P=0.009)$. There was an overall trend toward residual LV dysfunction in the group of patients with a dilated left ventricle in the initial echo $(18.8 \%$ versus $6.7 \%, P=0.3)$ as compared to a non-dilated left ventricle (Table 3).

\section{DISCUSSION}

The prevalence of PPCM in our institution in southern Israel was comparable to the prevalence reported in some centers in the United States, and the disease was less common than in other countries such as South Africa (1:1,000 births) or Haiti (1:300).1,14,15,34 The reasons for the difference in prevalence is probably related to ethnic as well as socio-economic factors. As mentioned, African descent is a consistent risk factor for the development of PPCM.6,7 In our cohort, there was a higher prevalence of the disease among Bedouin women as evidenced by the fact that this ethnic group made up almost $70 \%$ of patients diagnosed with PPCM, while constituting only about $40 \%$ of the overall maternal population according to local registries over the past few years. Moreover, the prevalence of PPCM was 
Table 1. Characteristics of Women with Peripartum Cardiomyopathy (PPCM).

\begin{tabular}{|l|c|c|c|}
\hline Characteristic & Bedouin $(\boldsymbol{n}=\mathbf{3 2})$ & $\begin{array}{c}\text { Non-Bedouin } \\
(\boldsymbol{n}=14)\end{array}$ & $\boldsymbol{P}$ Value \\
\hline Maternal age, mean (SD) & $30.4(6.9)$ & $31.2(6.7)$ & 0.690 \\
Gestational week, median (IQR) & $36.3(3.6)$ & $37.0(3.2)$ & 0.542 \\
Birth weight, g (SD) & $2,567(0.893)$ & $2,978(0.816)$ & 0.148 \\
Gestational number, median (IQR) & $5.0(1.0-8.0)$ & $2.5(1.0-6.2)$ & 0.244 \\
No. of live birth, median (IQR) & $5.0(1.0-7.0)$ & $2.5(1.0-5.2)$ & 0.343 \\
APGAR 1 min, median (IQR) & $9.0(5.0-9.0)$ & $9.0(8.7-9.0)$ & 0.400 \\
APGAR 5 min, median (IQR) & $10.0(9.2-10.0)$ & $10.0(10.0-10.0)$ & 0.179 \\
Diabetes mellitus, $n$ (\%) & $0(0.0)$ & $1(7.1)$ & 0.304 \\
Hypertension, $n$ (\%) & $0(0.0)$ & $0(0.0)$ & 1.000 \\
Obesity, $n$ (\%) & $4(12.5)$ & $2(14.3)$ & 0.869 \\
Dyslipidemia, $n(\%)$ & $0(0.0)$ & $1(7.1)$ & 0.304 \\
\hline
\end{tabular}

IQR, interquartile range.

Table 2. Echocardiographic Characteristics of Patients with PPCM.

\begin{tabular}{|l|c|c|}
\hline Characteristic & $\begin{array}{c}\text { First } \\
\text { Echocardiogram } \\
(\boldsymbol{n}=\mathbf{4 6 )}\end{array}$ & $\begin{array}{c}\text { Second } \\
\text { Echocardiogram } \\
(\boldsymbol{n}=46)\end{array}$ \\
\hline LV systolic function & None & $30(65.2)$ \\
$\quad$ Normal, $n(\%)$ & $16(34.8)$ & $9(19.6)$ \\
\hline Mild dysfunction, $n(\%)$ & $19(41.3)$ & $3(6.5)$ \\
Moderate dysfunction, $n(\%)$ & $11(23.9)$ & $4(8.7)$ \\
Severe dysfunction, $n(\%)$ & $7(15.2)$ & $0(0.0)$ \\
Regional hypokinesis, $n(\%)^{*}$ & $5(10.9)$ & $2(4.3)$ \\
Dilated LV, $n(\%)$ & $7(15.2)$ & $3(6.5)$ \\
Hypokinetic RV, $n(\%)$ & $7(15.2)$ & $2(4.3)$ \\
\hline Pulmonary HTN, $n(\%)$ & & \\
\hline
\end{tabular}

*Reference is global hypokinesis.

HTN, hypertension; LV, left ventricle; RV, right ventricle.

almost two-fold higher in Bedouin women than in the non-Bedouin population. Again, there may be a genetic base for this difference, and local customs may also influence these rates. In a country such as Israel, where there is universal access to health care, socio-economic factors seem to play a smaller role.

The lack of mortality cases evidenced in our study compared to global literature was a surprising finding. We tried to account for this effect by select- ing a more general diagnosis and actively reviewing echo results, to find cases that met our inclusion criteria. Another reason for the null mortality rate in our study might be the fact that we excluded patients presenting with another medical diagnosis that could explain the diminished LV systolic function such as severe sepsis or electrolyte imbalances; it is possible that these patients did suffer from severe PPCM that put them at increased risk for an adverse critical illness. Finally, as discussed before, 
Table 3. Characteristics of the First Echocardiogram in Patients With and Without Residual Peripartum Cardiomyopathy (PPCM).

\begin{tabular}{|c|c|c|c|}
\hline Characteristics & $\begin{array}{l}\text { Residual PPCM } \\
(n=16)\end{array}$ & $\begin{array}{l}\text { No Residual PPCM } \\
\qquad(n=30)\end{array}$ & $P$ Value \\
\hline Mean time between echo exams, days (SD) & $906(1,001)$ & $694(1,066)$ & 0.515 \\
\hline \multicolumn{4}{|l|}{ LV systolic function } \\
\hline Moderate or severe LV dysfunction, $n$ (\%) & $13(81.2)$ & $17(56.7)$ & 0.09 \\
\hline Moderate dysfunction, $n$ (\%) & $5(31.2)$ & $14(46.7)$ & NA \\
\hline Severe dysfunction, $n(\%)$ & $8(50.0)$ & $3(10.0)$ & NA \\
\hline Regional hypokinesis, $n(\%)^{*}$ & $2(12.5)$ & $5(16.7)$ & 1.000 \\
\hline Dilated LV, $n(\%)$ & $3(18.8)$ & $2(6.7)$ & 0.325 \\
\hline Hypokinetic RV, n (\%) & $2(12.5)$ & $5(16.7)$ & 1.000 \\
\hline Pulmonary HTN, $n(\%)$ & $1(6.2)$ & $6(20.0)$ & 0.394 \\
\hline
\end{tabular}

*Reference is global hypokinesis.

NA, not applicable.

the universal access to medical care might play a role in early diagnosis of the condition, thereby yielding better treatment strategies and closer medical follow-up for PPCM patients.

Some interesting findings arose from the comparison of patients who regained normal systolic LV function to those with residual systolic dysfunction on follow-up echo exams. The small number of cases prevented us from performing additional comparison between the two groups based on the severity of cardiac dysfunction. Of interest, women who had severe disease at presentation also had a lower chance of regaining normal LV function in comparison to those with a milder disease; the same was true for patients with dilated cardiomyopathy as compared to those with a non-dilated left ventricle. When adjusted to maternal age, the degree of LV dysfunction in the first echo remained an independent risk factor for long-term persistence of $\mathrm{LV}$ dysfunction. These findings add to the bulk of evidence that point to an association between the severity of LV systolic dysfunction and LV diameter at the time of PPCM diagnosis and the recovery of LV function. ${ }^{29-32,34}$ Nevertheless, it is important to note that baseline EF has limited sensitivity for prediction of improvement in individual patients, and it should not be used in selecting patients for more aggressive initial therapies. ${ }^{30}$

While much attention has been paid to $\mathrm{LV}$ characteristics, only recent studies have examined the importance of RV dysfunction as a potential prognostic factor in PPCM. 35 The incidence of RV hypokinesis in our study population was $15.2 \%$, while researchers in large prospective cohorts in Nigeria have reported rates of RV dysfunction ranging from $38 \%$ to $54 \%$ of women suffering from PPCM as well as an association between altered RV function and persistent LV dysfunction. ${ }^{36,37}$ Our lower percentage of RV injury is probably due to the fact that the main objective of these studies was to assess RV function specifically, leading to a more focused examination of the right ventricle than would be the case in a routine echo examination. As a result of our small sample size, we did not find an association between the presence of RV hypokinesis and persistence of $\mathrm{LV}$ dysfunction on follow-up, but this is a point that should be examined further.

Our study has some strong points and a few disadvantages. First of all, we performed a systematic search of all the patients who had received a diagnosis of cardiovascular disease during pregnancy for the past 11 years in our institution, and actively reviewed all echo results to be sure to include only patients who fit the PPCM criteria and to minimize the effect of selection bias. Secondly, our institution has an electronic database for obstetric and medical data that facilitated a more systematic search for the needed information. Even so, the number of cases was still too small to identify a statistical difference in most variables analyzed. Third, since our research was a retrospective cohort using an electronic database we had no access to detailed clinical com- 
plaints, signs, and symptoms. Patients were identified after PPCM diagnosis had already been added to our electronic database. Another disadvantage is that we did not search for outcomes in subsequent pregnancies. In addition, the management protocol for this research was left to the discretion of the treating physician due to the retrospective nature of the electronic database analysis. It should be emphasized that all patients underwent a follow-up echo that allowed for comparison between those who did and did not recover LV function. Finally, the complication rates (other than residual LV dysfunction) were not available from the database. It would have been thought-provoking to describe the rates for arrhythmias and thromboembolic events in our PPCM patients, but we were content with mortality rate, since death is the clinical endpoint of interest in these events.

\section{CONCLUSION}

Peripartum cardiomyopathy is a known although rare complication of late pregnancy and early puerperium, with outcomes varying from complete recovery of $\mathrm{LV}$ function to severe refractory congestive heart failure leading to heart transplant or even death. Our study has identified that the Bedouin population may be at increased risk for this condition and that a more severe degree of $\mathrm{LV}$ dysfunction is probably associated with a reduced chance for full recovery. Moreover, there is a growing need for investigation of the relationship between RV characteristics and PPCM.

\section{REFERENCES}

1. Pearson GD, Veille JC, Rahimtoola S, et al. Peripartum cardiomyopathy: National Heart, Lung, and Blood Institute and Office of Rare Diseases (National Institutes of Health) workshop recommendations and review. JAMA 2000;283:1183-8. Crossref

2. Hilfiker-Kleiner D, Kaminski K, Podewski E, et al. A cathepsin D-cleaved $16 \mathrm{kDa}$ form of prolactin mediates postpartum cardiomyopathy. Cell 2007;128:589. Crossref

3. Halkein J, Tabruyn SP, Ricke-Hoch M, et al. MicroRNA-146a is a therapeutic target and biomarker for peripartum cardiomyopathy. J Clin Invest 2013;123: 2143. Crossref

4. Patten IS, Rana S, Shahul S, et al. Cardiac angiogenic imbalance leads to peripartum cardiomyopathy. Nature 2012;485:333. Crossref
5. Elkayam U, Akhter MW, Singh H, et al. Pregnancyassociated cardiomyopathy: clinical characteristics and a comparison between early and late presentation. Circulation 2005;111:2050. $\underline{\text { Crossref }}$

6. Veille JC. Peripartum cardiomyopathies: a review. Am J Obstet Gynecol 1984;148:805. Crossref

7. Brar SS, Khan SS, Sandhu GK, et al. Incidence, mortality, and racial differences in peripartum cardiomyopathy. Am J Cardiol 2007;100:302-4. Crossref

8. Bello N, Rendon IS, Arany Z. The relationship between pre-eclampsia and peripartum cardiomyopathy: a systematic review and meta-analysis. J Am Coll Cardiol 2013;62:1715.

9. Homans DC. Peripartum cardiomyopathy. N Engl J Med 1985;312:1432. Crossref

10. Lampert MB, Hibbard J, Weinert L, et al. Peripartum heart failure associated with prolonged tocolytic therapy. Am J Obstet Gynecol 1993;168:493. Crossref

11. Mendelson MA, Chandler J. Postpartum cardiomyopathy associated with maternal cocaine abuse. Am J Cardiol 1992;70:1092. $\underline{\text { Crossref }}$

12. Sliwa K, Fett J, Elkayam U. Peripartum cardiomyopathy. Lancet 2006;368:687-93 Crossref

13. Elkayam U. Clinical characteristics of peripartum cardiomyopathy in the United States: diagnosis, prognosis and management. J Am Coll Cardiol 2011; 58:659-70. $\underline{\text { Crossref }}$

14. Sliwa K, Hilfiker-Kleiner D, Petrie MC, et al. Current state of knowledge on aetiology, diagnosis, management, and therapy of peripartum cardiomyopathy: a position statement from the Heart Failure Association of the European Society of Cardiology Working Group on peripartum cardiomyopathy. Eur J Heart Fail 2010;12:767-78. Crossref

15. Sliwa K, Damasceno A, Mayosi BM. Epidemiology and etiology of cardiomyopathy in Africa. Circulation 2005;112:3577. $\underline{\text { Crossref }}$

16. Sliwa K, Skudicky D, Bergemann A, et al. Peripartum cardiomyopathy: analysis of clinical outcome, left ventricular function, plasma levels of cytokines and Fas/APO-1. J Am Coll Cardiol 2000;35:701. Crossref

17. Goland S, Modi K, Bitar F, et al. Clinical profile and predictors of complications in peripartum cardiomyopathy. J Card Fail 2009;15:645. Crossref

18. McNamara DM, Elkayam U, Alharethi R, et al. Clinical outcomes for peripartum cardiomyopathy in North America: results of the IPAC study (Investigations of Pregnancy-Associated Cardiomyopathy). J Am Coll Cardiol 2015;66:905-14. $\underline{\text { Crossref }}$ 
19. Felker GM, Jaeger CJ, Klodas E, et al. Myocarditis and long-term survival in peripartum cardiomyopathy. Am Heart J 2000;140:785. Crossref

20. Felker GM, Thompson RE, Hare JM, et al. Underlying causes and long-term survival in patients with initially unexplained cardiomyopathy. N Engl J Med 2000;342:1077. $\underline{\text { Crossref }}$

21. Sliwa K, Forster O, Libhaber E, et al. Peripartum cardiomyopathy: inflammatory markers as predictors of outcome in 100 prospectively studied patients. Eur Heart J 2006;27:441-6. Crossref

22. Haghikia A, Podewski E, Libhaber E, et al. Phenotyping and outcome on contemporary management in a German cohort of patients with peripartum cardiomyopathy. Basic Res Cardiol 2013;108:366. Crossref

23. Murali S, Baldisseri MR. Peripartum cardiomyopathy. Crit Care Med 2005;33:S340. Crossref

24. Modi KA, Illum S, Jariatul K, et al. Poor outcome of indigent patients with peripartum cardiomyopathy in the United States. Am J Obstet Gynecol 2009;201: 171.e1. $\underline{\text { Crossref }}$

25. Harper MA, Meyer RE, Berg CJ. Peripartum cardiomyopathy: population-based birth prevalence and 7year mortality. Obstet Gynecol 2012;120:1013.

26. Kao DP, Hsich E, Lindenfeld J. Characteristics, adverse events, and racial differences among delivering mothers with peripartum cardiomyopathy. JACC Heart Fail 2013;1:409. Crossref

27. Cooper LT, Mather PJ, Alexis JD, et al. Myocardial recovery in peripartum cardiomyopathy: prospective comparison with recent onset cardiomyopathy in men and nonperipartum women. J Card Fail 2012;18:28. $\underline{\text { Crossref }}$

28. Pillarisetti J, Kondur A, Alani A, et al. Peripartum cardiomyopathy: predictors of recovery and current state of implantable cardioverter-defibrillator use. J Am Coll Cardiol 2014;63:2831. $\underline{\text { Crossref }}$
29. Goland S, Bitar F, Modi K, et al. Evaluation of the clinical relevance of baseline left ventricular ejection fraction as a predictor of recovery or persistence of severe dysfunction in women in the United States with peripartum cardiomyopathy. J Card Fail 2011; 17:426. Crossref

30. Blauwet LA, Libhaber E, Forster O, et al. Predictors of outcome in 176 South African patients with peripartum cardiomyopathy. Heart 2013;99:308. Crossref

31. Chapa JB, Heiberger HB, Weinert L, et al. Prognostic value of echocardiography in peripartum cardiomyopathy. Obstet Gynecol 2005;105:1303.

32. Sliwa K, Böhm M. Incidence and prevalence of pregnancy-related heart disease. Cardiovasc Res 2014;101:554-60. $\underline{\text { Crossref }}$

33. Ponikowski P, Voors AA, Anker SD, et al. 2016 ESC Guidelines for the diagnosis and treatment of acute and chronic heart failure: The Task Force for the diagnosis and treatment of acute and chronic heart failure of the European Society of Cardiology (ESC). Developed with the special contribution of the Heart Failure Association (HFA) of the ESC. Eur J Heart Fail 2016;18:891-975. Crossref

34. Carvalho A, Brandao A, Martinez EE, et al. Prognosis in peripartum cardiomyopathy. Am J Cardiol 1989; 64:540-2. $\underline{\text { Crossref }}$

35. Karaye KM. Right ventricular systolic function in peripartum and dilated cardiomyopathies. Eur J Echocardiogr 2011;12:372-4. $\underline{\text { Crossref }}$

36. Karaye KM, Lindmark K, Henein M. Right ventricular systolic dysfunction and remodelling in Nigerians with peripartum cardiomyopathy: a longitudinal study. BMC Cardiovasc Disord 2016;16:27. Crossref

37. Blauwet LA, Delgado-Montero A, Ryo K, et al. Right ventricular function in peripartum cardiomyopathy at presentation is associated with subsequent left ventricular recovery and clinical outcomes. Circ Heart Fail 2016;9;pii: eoo2756. $\underline{\text { Crossref }}$ 